\title{
Personalized Medicine: Impact on Patient Care in Genetic Counseling
}

\author{
Rebecca Nagy • Amy C. Sturm
}

Published online: 9 March 2013

(c) Springer Science+Business Media New York 2013

\begin{abstract}
With the completion of the Human Genome Project and rapid advances in biotechnology, including nextgeneration sequencing techniques, it is undeniable that the era of genomic medicine is upon us. There are numerous examples of how this information and technology is already being used in the diagnosis, treatment, and prevention of geneticbased disease. We review some of the recent highlights in the field and discuss how these advances will impact medicine and the delivery of care connected to genomic information to patients and their families.
\end{abstract}

Keywords Genomics · Genomic counseling · Whole exome sequencing · Whole genome sequencing - Genetic counseling

\section{Introduction}

Personalized medicine is a rapidly advancing field that uses a person's unique clinical, genetic, genomic, and environmental makeup to effectively diagnose, treat, and prevent

Rebecca Nagy and Amy Sturm contributed equally to this article.

\section{R. Nagy $(\bowtie) \cdot$ A. C. Sturm}

Division of Human Genetics, Wexner Medical Center, Ohio

State University, 2001 Polaris Parkway, Columbus, OH 43240,

USA

e-mail: rebecca.nagy@osumc.edu

A. C. Sturm

e-mail: amy.sturm@osumc.edu

A. C. Sturm

The Dorothy M. Davis Heart and Lung Research Institute, College of Medicine, The Ohio State University, Columbus, $\mathrm{OH}$, USA disease in an individualized manner. It has been made possible in part by the huge advances in genetic technologies over the past 10-15 years. Since 2003, the number of clinically available genetic tests has skyrocketed from several hundred to over 3,000. This was in large part due to the completion of the Human Genome Project in 2001 and concurrent advances in the ability to perform what is often referred to as high-throughput genomic sequencing-a rapid, affordable means of sequencing and analyzing large amounts of DNA sequence information. Two of the most commonly used tests are whole exome sequencing (WES) and whole genome sequencing (WGS). WES involves sequencing all of the exons, or coding regions, of a person's genome, whereas WGS sequences both the coding and the noncoding portions of the genome. As a direct result of these advances, clinicians are now able to order both of these tests in the clinical setting for roughly $\$ 4,000$ to $\$ 8,000$. The cost of sequencing will continue to drop precipitously over the next several years, and thus it will soon be more cost-efficient to perform WGS than to perform DNA Sanger sequencing for a single gene. As these discoveries make their way into mainstream medicine, there will be not only practical questions regarding implementation, but also ethical, legal, and societal challenges. How will genomic technologies change the delivery of health care? What are the risks, benefits, and limitations of these new technologies and how will we as a society handle these challenges? What tools and information are lacking in order for us to use this information to its greatest potential? What will our patients do with this information and will it improve lives? Many of these questions remain unanswered despite the fact that WGS has already arrived in the clinic. In this review we highlight some of the most recent examples of genomic medicine being used in the diagnosis and treatment of disease. We also provide a 
high-level overview of the challenges to implementation, potential solutions, and the impact of genomics on clinical practice.

\section{Discovery and Diagnosis-The Translation of WES/ WGS into the Clinic}

It is an exciting time to be providing clinical genetic and genomic medicine services, with one of the potential beneficial applications of WES/WGS technologies being that of an accurate diagnosis that ends the "diagnostic odyssey" for many patients and families. In the research setting, WES/WGS technologies have been used for Mendelian and complex disease gene discovery for several years $[1,2]$ and continue to be used to identify novel genetic causes of neurological disorders [3, 4], syndromic conditions [5, 6], and other varied phenotypes ranging from hereditary pancreatitis [7] to Stargardt disease [8], a form of early-onset macular degeneration. WES/WGS in patients with autism spectrum disorders has provided data regarding the significance of de novo mutations in the genetic risk of these conditions [9] and has also identified important regions of hypermutability [10]. Similarly, WES was recently applied to a group of 100 patients with intellectual disability (defined as an IQ below 50) and their unaffected parents and led to a definitive genetic diagnosis in $16 \%$ of them. These diagnoses may lead to improvements in treatment and offer parents both anticipatory guidance about their child's condition and information about recurrence risk in future pregnancies [11]. The past year bore witness to a movement of these genomic technologies from the research arena into the realm of clinically available diagnostic tests $[12,13]$, and success stories about the use of these technologies for clinical diagnosis have made their way into the popular press. Perhaps the most well-known and oft-cited example involves a male child with very severe inflammatory bowel disease where exome sequencing identified a novel mutation in the $\mathrm{X}$-linked inhibitor of the apoptosis gene. This was an unexpected finding as this gene had not previously been associated with inflammatory bowel disease, but it allowed specific medical therapy to be initiated using allogeneic hematopoietic progenitor cell transplant [14]. The patient had complete resolution of colitis. This is the power of personalized medicine.

Over the past year, multiple groups have published their experience using WES in the clinical setting and its ability to diagnose likely genetic, not clearly understood, conditions $[13,15 \bullet$ Need et al. [13] performed WES on a clinically representative sample of 12 parent-child trios where the child had congenital anomalies and/or intellectual disabilities presumed to be genetic, yet previous clinical tests including karyotype, chromosome microarray, and metabolic testing, among others, had been nondiagnostic. Their success rate was $50 \%$, with six out of 12 cases receiving a genetic diagnosis. Even though clear changes in treatment were not made in all cases, there was value in establishing a diagnosis for several of the cases, including the ability to provide specific medical screening recommendations, the need to avoid certain drugs, and recurrence risk information for the family. Also, Need et al. pointed out that although they did not perform a formal cost-benefit analysis, the argument can be made that WES approaches may be more cost-effective than sequencing one gene at a time or even ordering next-generation sequencing panels of genes, since prior to performing WES, more than $\$ 22,000$ was spent on laboratory tests for one of the trios. This study also presents several challenges in the clinical application of WES, including the difficulty in interpreting results in patients who do not have the typical clinical features associated with known Mendelian diseases, the current need for laboratory-based functional evaluation of de novo variants, the need for substantial manual examination of sequence information and candidate genes, and the complexity of genetic counseling when the phenotype is likely due to more than one genetic mutation. In another recent study, Dixon-Salazar et al. [15•] described the use of WES in a series of 118 probands with a diagnosis of pediatric-onset neurodevelopmental disease in which most known causes had been excluded. WES led to a corrected or new diagnosis in $27 \%$ of these individuals.

WGS technologies have also made their way into the realm of prenatal diagnosis and the neonatal intensive care unit (NICU). WGS technologies were used to complement standard cytogenetic analyses on an amniotic fluid sample in order to specifically discover disruption of the $\mathrm{CHD} 7$ gene, which causes CHARGE syndrome [16]. Although this prenatal diagnosis relied on amniocentesis, an invasive procedure with inherent risks, it has now been shown that WGS of a human fetus may soon be clinically feasible using noninvasive techniques. By the combination of WGS of both parents, genome-wide maternal haplotyping, and deep sequencing of maternal plasma DNA, the WGS of a human fetus was determined, noninvasively, at 18.5 weeks' gestation [17••]. Although this breakthrough has major ethical and societal implications, it also highlights areas where this technology can lead to improved prediction of inheritance patterns and recurrence rates and incorporation of new methods to detect genomic variation beyond single-nucleotide variants. Saunders et al. [18••] provided proof of concept for 2-day (approximately $50 \mathrm{~h}$ ) WGS to achieve a differential diagnosis of acutely ill neonates with suspected genetic disorders. Before the publication of this novel WGS protocol, clinical use of 
WGS using next-generation sequencing methods had taken at least 1 month. However, in the NICU and other acute care settings, a 1-month turnaround time may not meet that required for medical decision-making. To obtain these WGS results rapidly, the investigators developed several novel approaches that led to an accurate genetic diagnosis in a much shorter time. In two blinded, retrospective analyses, the team was able to correctly confirm known diagnoses using the rapid WGS approach; prospectively four of five affected individuals received definite or likely molecular diagnoses in approximately $50 \mathrm{~h}$. These studies not only show the power of genomics in diagnostic care but also demonstrate that some of the challenges in implementing these tools in a clinical setting can be overcome, making widespread use of genomic medicine in the clinic a much more tangible possibility.

\section{WES and WGS in Therapeutics}

\section{Treatment of Mendelian Disorders}

As WGS and WES move into the clinical arena, the resultant improvements in diagnostic approaches will likely lead to changes and/or improvements in treatment of genetic disease [19]. Although many inherited disorders are not amenable to treatment, the benefit of an early and accurate diagnosis can improve outcomes and in some cases lead to a lessening and/or complete relief of symptoms and/or avoidance of major complications. One such example was reported by Bainbridge et al. [20], who used WGS in 14-year-old fraternal twins who carried the diagnosis of 3,4-dihydroxyphenylalanine (dopa)-responsive dystonia (Mendelian Inheritance in Man \#128230). Doparesponsive dystonia is genetically heterogeneous and is a disease that can be inherited as either autosomal recessive or autosomal dominant. Standard treatment includes the use of L-dopa, a precursor of the neurotransmitter dopamine, which offers variable relief of symptoms. The patients in this report were diagnosed at age 5 years, after treatment with L-dopa had shown relief of major symptoms. However, both still experienced symptoms that disrupted daily functioning. In the twins, genetic testing was negative for two of the five genes known to be causative of dopa-responsive dystonia. WGS revealed that both twins were compound heterozygotes for missense mutations in the SPR gene, which had been previously implicated in dopa-responsive dystonia but for which clinical testing was unavailable at the time of their initial evaluation. Alterations in SPR are known to prevent regeneration of tetrahydrobiopterin, a cofactor for the production of dopamine and serotonin. Treatment for $S P R$-related dopa-responsive dystonia includes not only L-dopa therapy but also treatment with the serotonin precursor 5-hydroxytryptophan. As a result of the WGS results, both twins received 5-hydroxytryptophan therapy and showed marked improvement in symptoms.

Several groups have also reported their experience using WES in larger clinical series. In the work by Dixon-Salazar et al. [15•] summarized in the previous section, ten probands $(8 \%)$ were found to have disease-causing mutations in known genes that led to a change in their diagnosis and in some cases new therapeutic approaches [15•]. This included a sibship of four with early-onset intellectual disability and mild dysmorphic features. WES revealed a homozygous mutation in the $M A N 2 B 1$ gene, leading to the diagnosis of $\alpha$-mannosidosis (Mendelian Inheritance in Man 248500). This condition is treatable through hematopoietic stem cell transplant, something that would not have been considered previously in the affected individuals. In the work by Need et al. [13], six individuals were found to have previously undiscovered germline mutations that led to a new diagnosis [13]. For two of these individuals, the diagnosis resulted in new treatment and/or surveillance that would not have been previously recommended, including avoidance of medications that would potentially exacerbate symptoms. These early studies show the great promise of genomic medicine not only in the diagnosis but also in the treatment of genetic disease.

\section{Cancer Genomics and Targeted Therapeutics}

Advances in genetic and genomic technologies have changed the practice of oncology. Since the discovery in 2001 of the drug imatinib as a targeted tyrosine kinase inhibitor for the treatment of chronic myeloid leukemia, the number of targeted therapies in cancer and other conditions has continued to grow [21]. As of 2012, the FDA had reported over 110 marketed drugs that have pharmacogenetic biomarkers on their label, and over half of these are used in the treatment of various cancers [22]. Nextgeneration sequencing and array techniques have taken this field to a whole new level, given the ability to rapidly and affordably screen multiple tumors at the same time. It is likely that the list of targeted therapies will skyrocket over the next decade. High-throughput tumor analysis is now being used to improve tumor classification, assess the effect of first-line therapies on the genetic makeup of tumors, uncover genetic and epigenetic pathways not previously implicated in tumorigenesis, and identify the basis of drug sensitivity and resistance. Recently, Iyer et al. [23] used WGS to identify a new biomarker for sensitivity to the drug everolimus in bladder cancer. They studied the genome of a patient with metastatic bladder cancer who had achieved a durable and ongoing complete response to everolimus, an inhibitor of mammalian target of rapamycin 
complex 1. WGS of the patient's primary tumor and blood showed a number of mutations in the tumor, including a two-base-pair deletion in the TSC1 gene and a nonsense mutation in the NF2 gene. Sequencing of an additional 96 high-grade bladder cancers showed that $5.2 \%(5 / 96)$ also had TSC1 mutations, indicating that this is a commonly mutated gene in bladder tumors. Analysis of an additional 13 bladder cancer patients who had received everolimus in the same clinical trial showed three additional tumors with somatic mutations in TSC1. When compared with those patients with tumors not carrying TSC1 mutations, patients with TSC1-mutant tumors received everolimus therapy for longer and had significantly improved times to recurrence, suggesting that the presence of a TSC1 mutation in the tumor is predictive of a better response to the drug everolimus. This study is an example of using WGS to determine which patients might be more sensitive to a specific chemotherapy agent, and in turn might have a better response to treatment. Roychowdhury et al. [24] used several high-throughput techniques to conduct a pilot study of four patients with advanced and refractory cancer who were under consideration for clinical trials. They performed multiple tests, including WGS and WES of patient blood and tumor samples. A panel of experts gathered to review the findings and determine which, if any, genomic changes might be amenable to targeted therapy. Two of the four patients had genetic alterations that could potentially be targeted with currently available therapies. In one patient, WGS/WES was able to explain why the patient had not responded to a previous chemotherapy agent, and also discovered mutations in two other genes that could be targeted using different drugs. Because of the narrow inclusion criteria for studies using such agents, which are often based on cancer type rather than the genetic makeup of the tumor, this patient was not eligible for any of the currently available clinical trials using these agents. This particular study underscores the need to move toward molecular-based inclusion and exclusion criteria for clinical trials. It also highlights the challenges that might slow implementation of genomic sequencing in oncology care.

\section{Genomic Counseling-Future Challenges and Potential Solutions}

With such technical advances on the near horizon and the vast potential of genomic medicine in the improved diagnosis and treatment of disease, it is likely that the number of health care practitioners using genetic and genomic testing for their patients will significantly increase over the next 5-10 years. However, several studies have shown that the majority of nongenetics providers rate themselves as having limited knowledge of genetics and do not feel equipped to correctly order and interpret the results of
Table 1 Similarities and differences between genetic and genomic testing and counseling

\begin{tabular}{|c|c|}
\hline Similarities & Differences \\
\hline $\begin{array}{l}\text { Need for extensive phenotyping } \\
\text { via collection of medical and } \\
\text { family history }\end{array}$ & $\begin{array}{l}\text { Time required for clinical } \\
\text { incorporation and effect on } \\
\text { reimbursement }\end{array}$ \\
\hline Genetic risk assessment & $\begin{array}{l}\text { Pretest counseling and informed } \\
\text { consent process }\end{array}$ \\
\hline $\begin{array}{l}\text { Translating complex genetic } \\
\text { information for patients and } \\
\text { health care providers }\end{array}$ & Unanticipated, incidental findings \\
\hline Familial implications & $\begin{array}{l}\text { Depth and breadth of information } \\
\text { discussed during genomic } \\
\text { counseling (possible multitude } \\
\text { of results and conditions verses } \\
\text { one test result for one } \\
\text { condition) }\end{array}$ \\
\hline Personal utility of information & $\begin{array}{l}\text { Dealing with information } \\
\text { overload and how to store, } \\
\text { update, and deliver data over } \\
\text { time }\end{array}$ \\
\hline Ethical, legal, societal issues & Turnaround time for results \\
\hline
\end{tabular}

genetic testing $[25,26]$. One solution to this problem is the use of health care providers with expertise in genetics who can serve as critical components of the health care team. Genetic counselors are well-suited to fill this need, given their training and expertise in genetics and genomics, risk assessment, and communication. Genetic counselors have been delivering genetic services for over 30 years and have already become an integral part of genomic medicine research and clinical care [27•, 28-33].

Currently, there is a paucity of data on the delivery of genomic counseling services, and most reports are based on a single institution's anecdotal experience and/or expert opinion [27•, 28, 34]. Although limited in scope, these reports shed light on some of the similarities and differences between genetic and genomic counseling (Table 1) and provide key insights. One report also offers practical recommendations for the provision of genomic counseling, including measures such as case preparation to help concentrate efforts on the patient's most significant medically actionable results, the use of publically available databases to evaluate genomic risk variants, the use of electronic educational tools both during the counseling session and after the session for continuing patient and provider education, the identification and/or development of novel visual aides to facilitate discussion of genomics concepts, and the use of teachable moments to provide the patient with a foundation of knowledge that may be used time and again for continued understanding and interpretation of genomic testing results [28]. These recommendations will likely evolve over time as more research is performed and experience is gained in the delivery of genomic counseling services. 
Research on the topic of genomic counseling completed to date also suggests that although the fundamental elements of a genomic counseling session will mostly be the same as those provided during "traditional" genetic counseling, there will likely be key differences in the breadth of discussion and the need for ongoing follow-up. For example, an imperative component of genomic counseling in both the research setting and the clinical setting is a thorough informed consent discussion, including explanations of current limitations of variant interpretation, as well as the overall categories of results that may or may not be disclosed [2]. The Presidential Commission for the Study of Bioethical Issues [35•*] recently published a timely report on privacy and progress in WGS, including topics and recommendations related to consent, data security and access, specimen sharing, privacy regulations, and legal protections regarding WGS data. Detailed consent and education for patients and families are necessary since interrogation of a patient's genome will likely provide incidental findings completely unrelated to the initial impetus for sequencing the genome of the individual in the first place. This could include medically actionable variants in genes associated with cancer predisposition syndromes and/or differential responses to medications and carrier status for recessive conditions. However, variants in genes associated with currently nonpreventable, nontreatable conditions as well as variants of unknown clinical significance will also be identified. Considerable questions regarding what should and should not be reported have therefore been raised. In fact, the National Human Genome Research Institute recently awarded seven grants totaling $\$ 5.7$ million aimed at these exact questions relating to "return of results." A recent study of 16 clinical genetics and/or molecular medicine specialists evaluated concordance for return of results to the ordering physician if they were found incidentally through WGS [36]. There was unanimous agreement on disclosure of 21 conditions within the realm of adult patients/known pathogenic mutations (e.g. long QT syndrome, multiple endocrine neoplasia type 1); however, specialists disagreed more when the patient was a child and for conditions where medical intervention is not currently available. One approach may be to release genomic information over time, as more is learned about the biological significance of certain genetic variants and/ or as the patient reaches a stage in life where the genomic information may be important for his/her medical management [37]. A "binning" system has also been proposed, which would separate clinically actionable results from WGS into a different category from those that are clinically valid yet not directly actionable and, further, from those that have unknown or no clinical significance [38]. In this way, return of results may be made scalable even at the public health level and this approach may also serve to avoid overwhelming patients and their physicians with large amounts of data that may not currently be meaningful. Interestingly, almost all those queried in a group of sequential participants in the NIH ClinSeq study regarding intention to learn their genome sequencing results indicated they would want the results, most often, for disease prevention, with attitudes rating the highest for medically actionable and carrier status results [29]. Ongoing incorporation and application of WGS information will be best undertaken using a team approach; a landmark article describing clinical assessment using WGS data for one patient cited hundreds of hours spent interpreting data by a team including a clinical geneticist, a genetic counselor, a bioinformatician, a genetic cardiologist, an internal medicine specialist, a pharmacogenomics specialist, and others [39].

\section{Conclusion}

It is undeniable that the future of genomic medicine is now, with its promise already witnessed in multiple clinical settings across the human life span. However, many questions, challenges, and potential barriers to effective implementation remain, including the availability of an adequately sized "genomic workforce," accurate and reliable genomic reference sequence databases, privacy of genomic information, bioinformatics capabilities, including genomic-friendly electronic medical record systems and parallel clinical decision support applications, and genomics education for health care professionals and the lay public. These challenges, along with the concomitant ethical, legal, and social issues surrounding genomics are complex and multilayered. Ongoing and future research will be critical to ensure that genomic technologies are used to their greatest potential in patient care, including a specific research agenda related to genomic counseling issues in clinical implementation.

Disclosure R. Nagy declares no conflict of interest and A. Sturm declares no conflict of interest.

\section{References}

Papers of particular interest, published recently, have been highlighted as:

- Of importance;

•- Of major importance

1. Ng SB, Turner EH, Robertson PD, et al. Targeted capture and massively parallel sequencing of 12 human exomes. Nature. 2009;461:272-6.

2. Bamshad MJ, Ng SB, Bigham AW, et al. Exome sequencing as a tool for Mendelian disease gene discovery. Nat Rev Genet. 2011;12:745-55. 
3. Rosewich $\mathrm{H}$, Thiele $\mathrm{H}$, Ohlenbusch A, et al. Heterozygous de-novo mutations in ATP1A3 in patients with alternating hemiplegia of childhood: a whole-exome sequencing gene-identification study. Lancet Neurol. 2012;11:764-73.

4. Bras J, Guerreiro R, Hardy J. Use of next-generation sequencing and other whole-genome strategies to dissect neurological disease. Nat Rev Neurosci. 2012;13:453-64.

5. Sarig O, Nahum S, Rapaport D, et al. Short stature, onychodysplasia, facial dysmorphism, and hypotrichosis syndrome is caused by a POC1A mutation. Am J Hum Genet. 2012;91:337-42.

6. Cabral RM, Kurban M, Wajid M, et al. Whole-exome sequencing in a single proband reveals a mutation in the CHST8 gene in autosomal recessive peeling skin syndrome. Genomics. 2012;99:202-8.

7. LaRusch J, Barmada MM, Solomon S, Whitcomb DC. Whole exome sequencing identifies multiple, complex etiologies in an idiopathic hereditary pancreatitis kindred. JOP. 2012;13:258-62.

8. Strom SP, Gao YQ, Martinez A, et al. Molecular diagnosis of putative Stargardt disease probands by exome sequencing. BMC Med Genet. 2012;13:67.

9. O'Roak BJ, Deriziotis P, Lee C, et al. Exome sequencing in sporadic autism spectrum disorders identifies severe de novo mutations. Nat Genet. 2011;43:585-9.

10. Michaelson JJ, Shi Y, Gujral M, et al. Whole-genome sequencing in autism identifies hot spots for de novo germline mutation. Cell. 2012;151:1431-42.

11. de Ligt J, Willemsen MH, van Bon BW, et al. Diagnostic exome sequencing in persons with severe intellectual disability. N Engl J Med. 2012;367:1921-9.

12. Mefford HC. Diagnostic exome sequencing-are we there yet? N Engl J Med. 2012;367:1951-3.

13. Need AC, Shashi V, Hitomi Y, et al. Clinical application of exome sequencing in undiagnosed genetic conditions. J Med Genet. 2012;49:353-61.

14. Worthey EA, Mayer AN, Syverson GD, et al. Making a definitive diagnosis: successful clinical application of whole exome sequencing in a child with intractable inflammatory bowel disease. Genet Med. 2011;13:255-62.

15. - Dixon-Salazar TJ, Silhavy JL, Udpa N et al. Exome sequencing can improve diagnosis and alter patient management. Sci Transl Med 2012;4:138ra178. This work is one of the first to use WES in a relatively large series of patients in the clinical setting. It highlights the potential benefits of WES in the diagnosis and treatment of rare Mendelian diseases, as well as the challenges in clinical implementation.

16. Talkowski ME, Ordulu Z, Pillalamarri V, et al. Clinical diagnosis by whole-genome sequencing of a prenatal sample. $\mathrm{N}$ Engl $\mathrm{J}$ Med. 2012;367:2226-32.

17. • Kitzman JO, Snyder MW, Ventura M et al. Noninvasive whole-genome sequencing of a human fetus. Sci Transl Med 2012;4:137ra176. This was the first work to use both WGS and "cell-free" capture of fetal DNA from the mother's blood to genotype a fetus.

18. • Saunders CJ, Miller NA, Soden SE et al. Rapid whole-genome sequencing for genetic disease diagnosis in neonatal intensive care units. Sci Transl Med 2012;4:154ra135. These authors developed several novel approaches for sample preparation, bioinformatic analysis, and clinical correlation that allowed WGS results to be obtained rapidly. This approach was used prospectively in five affected NICU patients, four of whom received definite or likely molecular diagnoses within approximately $50 \mathrm{~h}$.

19. Cordero P, Ashley EA. Whole-genome sequencing in personalized therapeutics. Clin Pharmacol Ther. 2012;91:1001-9.

20. Bainbridge MN, Wiszniewski W, Murdock, et al. Whole-genome sequencing for optimized patient management. Sci Transl Med. 2011;3:87.
21. Druker BJ, Talpaz M, Resta DJ, et al. Efficacy and safety of a specific inhibitor of the BCR-ABL tyrosine kinase in chronic myeloid leukemia. N Engl J Med. 2001;344:1031-7.

22. Institute of Medicine. Genome-based therapeutics: targeted drug discovery and development: workshop summary. Washington: National Academies Press; 2012.

23. Iyer G, Hanrahan AJ, Milowsky MI, et al. Genome sequencing identifies a basis for everolimus sensitivity. Science. 2012; 338:221.

24. Roychowdhury S, Iyer MK, Robinson, et al. Personalized oncology through integrative high-throughput sequencing: a pilot study. Sci Transl Med. 2011;3:111-21.

25. Giardiello FM, Brensinger JD, Petersen GM, et al. The use and interpretation of commercial APC gene testing for familial adenomatous polyposis. N Engl J Med. 1997;336:823-7.

26. Wideroff L, Vadaparampil ST, Greene MH, et al. Hereditary breast/ovarian and colorectal cancer genetics knowledge in a national sample of US physicians. J Med Genet. 2005;42:749-55.

27. - O'Daniel JM, Lee K. Whole-genome and whole-exome sequencing in hereditary cancer: impact on genetic testing and counseling. Cancer J. 2012;18:287-92. Reviews the currently available next generation sequencing technology and provides an overview of the challenges of genomic counseling for wholegenome and whole exome sequencing results.

28. Sturm AC, Manickam K. Direct-to-consumer personal genomic testing: a case study and practical recommendations for "genomic counseling". J Genet Couns. 2012;21:402-12.

29. Facio FM, Eidem H, Fisher T, et al. Intentions to receive individual results from whole-genome sequencing among participants in the ClinSeq study. Eur J Hum Genet. 2013;21:261-5.

30. Dewey FE, Chen R, Cordero SP, et al. Phased whole-genome genetic risk in a family quartet using a major allele reference sequence. PLoS Genet. 2011;7:e1002280.

31. Johnston JJ, Rubinstein WS, Facio FM, et al. Secondary variants in individuals undergoing exome sequencing: screening of 572 individuals identifies high-penetrance mutations in cancer-susceptibility genes. Am J Hum Genet. 2012;91:97-108.

32. Facio FM, Brooks S, Loewenstein J, et al. Motivators for participation in a whole-genome sequencing study: implications for translational genomics research. Eur J Human Genet. 2011; 19:1213-7.

33. O'Daniel J, Haga SB. Public perspectives on returning genetics and genomics research results. Public Health Genomics. 2011; 14:346-55.

34. Ormond KE, Wheeler MT, Hudgins L, et al. Challenges in the clinical application of whole-genome sequencing. Lancet. 2010;375:1749-51.

35. • Presidential Commission for the Study of Bioethical Issues. Privacy and progress in whole genomic sequencing. Washington: Presidential Commission for the Study of Bioethical Issues; 2012. p. 154. This report reviews the public benefits anticipated from WGS research and the potential risks to the privacy of individuals. It provides a set of recommendations and policies to protect an individual's privacy while maximizing the potential for scientific and medical progress.

36. Green RC, Berg JS, Berry GT, et al. Exploring concordance and discordance for return of incidental findings from clinical sequencing. Genet Med. 2012;14:405-10.

37. Sharp RR. Downsizing genomic medicine: approaching the ethical complexity of whole-genome sequencing by starting small. Genet Med. 2011;13:191-4.

38. Berg JS, Khoury MJ, Evans JP. Deploying whole genome sequencing in clinical practice and public health: meeting the challenge one bin at a time. Genet Med. 2011;13:499-504.

39. Ashley EA, Butte AJ, Wheeler MT, et al. Clinical assessment incorporating a personal genome. Lancet. 2010;375:1525-35. 